\title{
Table Lifting Mechanism Design for Medical Surgery Application base on Human Force Driven
}

\author{
Lukas Novak', Marek Penhaker ${ }^{2, *}$ and Ondrej Frantisek ${ }^{1}$ \\ 'Department of Mechanics, VSB - TU Ostrava, Faculty of Mechanical Engineering, Ostrava, 17. Listopadu 15, 708 33, Czech Republic \\ ${ }^{2}$ Department of Cybernetics and Biomedical Engineering, VSB - TU Ostrava, Faculty of Electrical Engineering and Computer Science, Ostrava, 17. Lis- \\ topadu 15, 708 33, Czech Republic
}

\begin{abstract}
The paper deals with a concept of lifting mechanisms for surgery tables powered by human force only. The issue is to design a simple surgery table without any use of electricity or hydraulic equipment. The lifting mechanism of the table is based purely on mechanical components and is driven by a human power. Force and kinematical relation was stated and evaluated for medical and veterinary usage of surgery tables. Two concepts of the lifting mechanism are introduced and investigated.
\end{abstract}

Keywords: lifting mechanism; table; surgery; medical, human powered; design

\section{Introduction}

The idea of a surgery table based upon mechanical components came up from the hospital background. Nowadays electrical or hydraulic operating tables are used in common operation. But these designs have several disadvantages. Let us discuss disadvantages of two current systems of table driving: electrical and hydraulic tables. [1-3]

Electrical surgery tables are noisy, heavy and non-manipulable depend on a source of electricity and occupy lot of space. Because of the weight and dimensional demands these have to be anchored deep in floor so a room situated under the surgery room is occupied as well. [4, 5]

Hydraulic systems bring also high demands for space and dangerous of liquid leakage, are expensive, need often and regular maintain. And the most crucial point: there has to be a source of pressurized medium.

The described problematic issues of electrical and hydraulic tables drive attention on a concept of surgery table using just mechanical components and driven by surgeon's foot. Mechanical table would be independent of any sources, easy manipulable, lightweight and low-cost. So the device would be optimal for hard conditions or veterinary purposes. [6-8]

It is therefore evident that these demanding requirements and behavior does not meet the parameters of each type. Nowadays we can find many companies, both foreign and domestic, that the issue of manufacturing operating tables dealing with. For a brief orientation, I can cite several major companies that have production, innovation, production and operating tables many years of experience and will help us to navigate in the current menu operating table. Among the leaders in the field include Trumpf, Nimotech, RAMED or Selet. When we look at their menu, we 
find that is currently most used operating tables, which are used for lifting and hydraulic equipment, or are driven by an electric motor. [9-12]

\subsection{Lifting Mechanism Requirements}

Indispensable equipment of each operating room is the operating table on which to place all the acts, and for which doctors must spend several hours to save lives. It is therefore extremely important to work with such a surgeon's table for the simplest, most convenient, and its design parameters that enable easy access operated on the injured areas. One of the most important parts of the table is the lifting mechanism. It must meet a number of technical requirements:

- It have to be seamlessly support the weight of the operated, but also its own weight operating table (approximately $220 \mathrm{~kg}$ ). The material used should therefore be as light as possible, yet strong, durable and must meet hygiene requirements.

- Emphasis is also placed on the lift characteristics of the table lifting mechanism should be easy to use and effortlessly. Smallest lift height of the table should match the height of the mobile deck chairs on which the patient is transported into the operating room from which is further transmitted to the device itself (about $600 \mathrm{~mm}$ ). The maximum stroke is limited to individual needs, a doctor himself so long for him and the work was not onerous nor causing eg. Back pain (about $1200 \mathrm{~mm}$ ). The actual stroke, but also the lowering should take place as smoothly as possible, because of patient comfort, but also for safety reasons, which occur e.g. in damaged spinal injured. On the other hand, a movement of the table should be fast enough.

- The design of the lifting mechanism should be as uncomplicated as possible and independent power sources. Another condition is a low noise, due to the nature of this mechanism.

- Production costs should be minimized.

- Lifting process should be smooth but also fast enough and safe. [13, 14]

The aim of this work will therefore propose possible variants of the lifting mechanism operating table, which will only work on mechanical principles. Lifting will be made to supply input works as a doctor pedaling on the pedal. The force will be using the individual members of the mechanism is transferred from a treadle pedal is reduced to a minimum and further transformed to allow the lifting itself.
The fundamental function of each surgery table is lifting of a body in vertical direction. The paper deals with a design of simple table which enables only this motion. Other motions like rotating of a body and so on are not examined.

\section{Kinematic and power analysis and synthesis of mechanisms}

Let us investigate a relation between input mechanical work of a surgeon's foot and output mechanical work of a lifted body. If we consider the transmission ratio of the lifting mechanism is constant or slightly varying, and then we can pronounce the input mechanical work of foot is equal to output mechanical work of lifted body, see scheme on Figure 1. Then, the mechanical work balance is as follows:

$F_{s} \cdot L \cdot \Delta \gamma=G \cdot \Delta h$,

where: FS - force of the surgeon's foot $[\mathrm{N}]$, $L$ - distance between the foot and joint of crank $2[\mathrm{~mm}], \Delta \gamma$ - angle of tread [-], G - the weight of body [N], $\Delta \mathrm{h}$ - lifted distance of the body [N].

Now we can evaluate relations between the quantities introduced in equation 1 . We consider the force of the surgeon's foot is $250 \mathrm{~N}$, the angle of tread is $30^{\circ}$, the distance $L$ is $350 \mathrm{~mm}$, and weight for the medical purposes is $2200 \mathrm{~N}$ and for veterinary purposes $1000 \mathrm{~N}$. After substituting the values to equation 1 , we get the magnitude of lifted distance $\Delta h$ per one tread. The lift $\Delta h$ is 21 $\mathrm{mm}$ for medical purposes and $46 \mathrm{~mm}$ for veterinary purposes. [15]

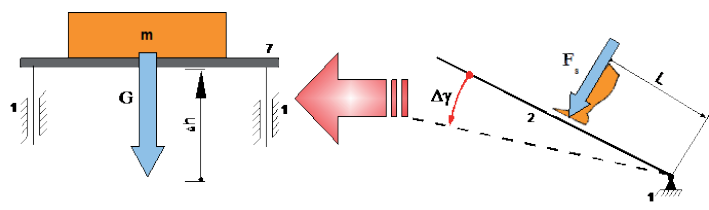

Fig. 1: Example of Mechanical Work of Lifted Body.

Unfortunately the value of the lift per one tread $21 \mathrm{~mm}$ is too low in case of medical purposes. A surgeon would have to tread too many times to lift the body. On the other hand the value of the lift $46 \mathrm{~mm}$ is found optimal in case of veterinary purposes. The author came to conclusion, the idea of the surgery table driven by surgeon's leg is reasonable mainly for veterinary usage. [16-19] 


\section{Mechanism based on system of pulleys}

The concept of the lifting mechanism is based on the system of pulleys. The kinematical scheme of the design is shown on the figure 2. The man force steps on crank 2 . The motion is transferred from crank 2 to rod 3 . The connection between the rod 3 and part 4 is realized as ratchet 3 and ratchetwheel 4 . The gear wheel of part 4 transfers rotation to part 5. Motion of the foot is transferred to rotation of part 4 . Rotation of part 4 is transmitted to part 5 by a gear connection. The smaller wheel of part 5 presents the wire strand drum. The wire form the drum 5 goes over the top pulley 6 to pulley 8 and then is anchored to the table frame 1 . Pulley 6 is used just to change the direction of wire motion. Pulley 8 performs general plane motion and is connected to the platform 7 so the pulley 8 lifts the platform 7 with the weight.

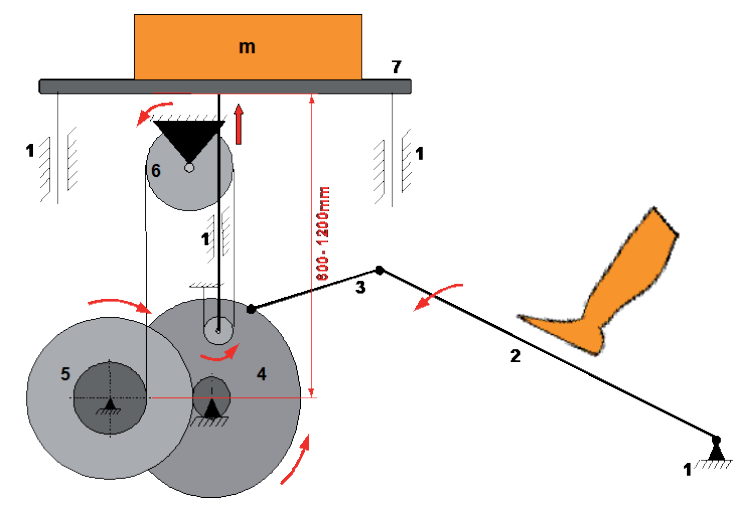

Fig. 2: Example of Mechanism Based on System of Pulleys.

Tab.1: The features of mechanism based on system of pulleys.

\begin{tabular}{|l|l|}
\hline Advantages & Disadvantages \\
\hline Structurally simple & Risk of vibration \\
\hline $\begin{array}{l}\text { Low friction } \rightarrow \text { therefore } \\
\text { higher efficiency }\end{array}$ & Need of maintenance \\
\hline Low built-in unit & \\
\hline
\end{tabular}

Transmission function defining kinematical relations and force relations were determined. Because of the paper scope the relations are not described here. There are two important conclusions of the investigation. The dimensions fitting the parameter given in equation 1 are feasible so the mechanism can be placed under the table. The second remark the mechanism transmission ratio is very slightly varying is satisfied for the reasonable setting of the lengths of parts number 2 and 3 and position of the joint between the frame 1 and crank 2 .

\subsection{Calculation of lifting dependence}

\subsubsection{Determination of the rotation gear 4}

Since this scheme is the same as in Fig. 3. 11, we can write the same equation as given in chapter 3. 3:

$l_{1}=l_{k} \cdot \cos \gamma+l_{o} \cdot \cos \beta+r_{4 v} \cdot \cos \alpha$

$l_{2}=l_{k} \cdot \sin \gamma+l_{0} \cdot \sin \beta+r_{4 v} \cdot \sin \alpha$

Now we know the angle of rotation of the gear 4, which again we have calculated numerically, we can determine the perimeter track, which executes a small gear 4 meters at its rotation as:

$s_{4 m}=\alpha \cdot r_{4 m}$

Fixing rotation member no. 6: Diagram of the rotation of the idler gear is shown in FIG. 4. 10. Knowing the circumferential path of the wheel $4 \mathrm{~m}$, the intermediate wheel perform the same distance, and we can therefore write the relation:

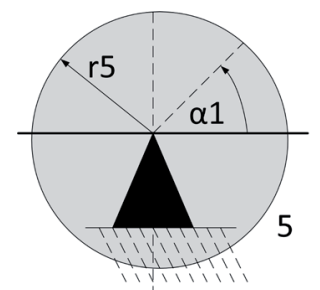

Fig. 3: Scheme rotation idler gear.

$s_{5}=s_{4 m}$

\subsubsection{Determination of turning gears 6}

Diagram of rotation is the same as in Fig. 3. 12 in Chapter \# 3. 3. Since the known circumferential path of the intermediate gear 5 , and known size of the path traveled by the large gear $6 \mathrm{v}$, because this value is the same. For this movement, we can determine the angle of rotation $\alpha 1$ of gear and then the size of the perimeter path of the small wheel 6 meters as:

$s_{6 v}=s_{5}$ 
$\alpha_{1}=\frac{S_{6 v}}{r_{6 v}}$

$s_{6 m}=\alpha_{1} \cdot r_{6 m}$

\subsubsection{Determination of circumferential tracks fixed pulley 7}

As for the drum 6 meters wound wire, which is further wound on the stationary pulley 7 , will shift that drum 6 m executes identical to traverse a fixed pulley seventh Thus we can write:

$s_{7}=s_{6 m}$

\subsubsection{Determining the perimeter track free pulley 8}

Length by which the cable is wound on a stationary pulley 7 , will be the same for winding loose roller 8 . Since the mere idle roller 8 held a general planar motion, will shift rod 9 identical. From Fig. 4. 11 can be readily seen that the velocity in the cable, which leads from the fixed pulley 7 is twice the speed of Wednesday loose positives 8 . This same applies to the distance of displacement, and therefore we can write equation:

$s_{8}=\frac{s_{7}}{2}$
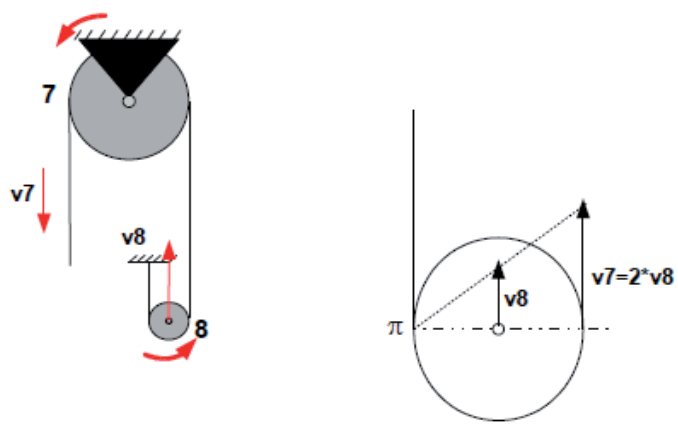

Fig. 4: Diagram speed at idle roller 8.

Set feed rods 9 and 10 table top: Due feed free rollers 8 will feed attached to a rigid rod 9 and the actual table top 10 . Since the distance is the same, we can write:

$s_{10}=s_{9}=s_{8}$

The resulting equation for the displacement of the plate we get when in equation (1) we substitute equation (10) to (4): $s_{10}=\frac{\frac{\alpha \cdot r_{4 m}}{r_{6 v}} \cdot r_{6 m}}{2}$

Like the pedal force and displacement is half compared to the values in the first shift mechanism. Table 2 below, shows the values of pedal force and stroke position of the operating table plate. Assuming that the surgeon will begin pedal crank 2 from the initial position $\gamma=30^{\circ}$ to the final $\gamma=10^{\circ}$, the resulting displacement equal to the difference S7 positioning plates in these positions. For parameters $m_{d}=220 \mathrm{~kg}, m_{\text {tyce }}=2 \mathrm{~kg}, m k_{1}=0,5 \mathrm{~kg}$, $l_{1}=500 \mathrm{~mm}, l_{2}=0 \mathrm{~mm}, l_{k}=400 \mathrm{~mm}, l_{0}=250 \mathrm{~mm}$, $r_{4 v}=160 \mathrm{~mm}, r_{4 m}=60 \mathrm{~mm}, r_{6 v}=80 \mathrm{~mm}, r_{6 m}=30 \mathrm{~mm}$.

$s_{10}=30,256-24,183=6,073 \mathrm{~mm}$

Tab. 2: Size pedal force to shift by changing the angle $\gamma$.

\begin{tabular}{|l|l|l|}
\hline$\gamma\left[^{\circ}\right]$ & $F_{s}[\mathrm{~N}]$ & $S_{10}[\mathrm{~mm}]$ \\
\hline 30 & 229,723 & 24,183 \\
\hline 25 & 213,033 & 25,949 \\
\hline 20 & 195,894 & 27,582 \\
\hline 15 & 171,605 & 29,057 \\
\hline 10 & 121,449 & 30,256 \\
\hline
\end{tabular}

\subsection{Sensitivity charts}

In the figures, see below, shows sensitivity graphs showing the dependency of the offset plate on the selected dimensions: $m_{d}=220 \mathrm{~kg}, m_{\text {tyce }}=2 \mathrm{~kg}, m k_{1}=$ $0,5 \mathrm{~kg}, l_{1}=500 \mathrm{~mm}, l_{2}=0 \mathrm{~mm}, l_{k}=400 \mathrm{~mm}, l_{0}=250 \mathrm{~mm}$, $r_{4 v}=160 \mathrm{~mm}, r_{4 m}=60 \mathrm{~mm}, r_{6 v}=80 \mathrm{~mm}, r_{6 m}=30 \mathrm{~mm}$.

Figure 4 shows the final stroke of the plate of the operating table 10, depending on the length of the handle 2. It can be seen that with increasing length of the handle 2 increases and resulting stroke.

\section{Optimization}

Optimization performed under the assumption that the surgeon will pedal crank $2=\gamma$ in the range of $30-10^{\circ}$. Furthermore, equations determine the initial values for the sighting angle $\alpha=114^{\circ}$ and $\beta=29^{\circ}$.

\subsection{Target function}

The target function, which we want to maximize, define the relation as:

$s=\frac{\frac{\alpha \cdot r_{4 m}}{r_{6 v}} \cdot r_{6 m}}{2}$ 

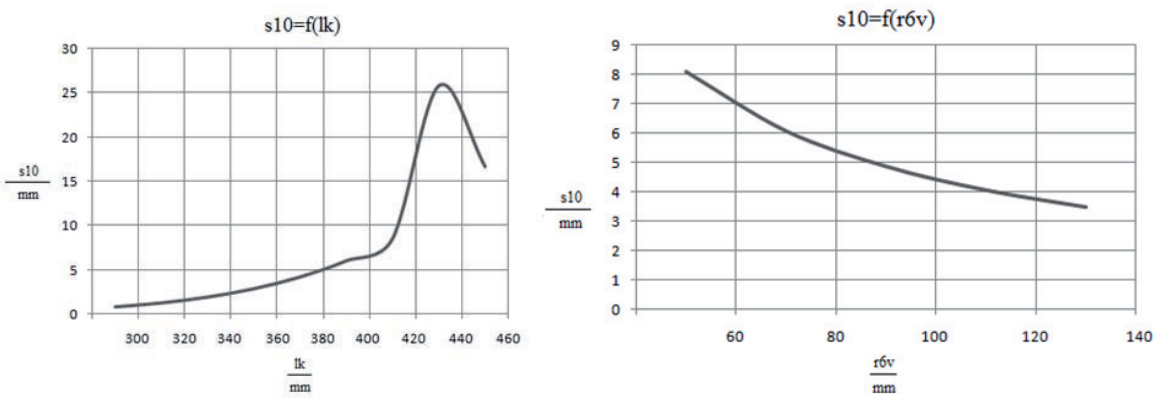

Fig. 5: Dependence on the length of the feed crank 2 (left), graph of the displacement member on the radius 7 (right).

\subsection{Tuning parameters}

For this alternative solution we will optimize the following parameters, which we defined as a vector of tuning parameters:

$$
z=\left[l_{k}, l_{o}, l_{1}, l_{2}, r_{4 v}, r_{4 m}, r_{6 v}, r_{6 m}\right]
$$

Stroke or pedal Force on the dimensions of the rollers depend at all, because their parameters can be selected as $r 7=80 \mathrm{~mm}$ and $r 8=60 \mathrm{~mm}$. Likewise, the diameter of the idler wheel has no force or effect on stroke and vote him as $r 7=70 \mathrm{~mm}$.

\subsection{Limitations}

Of Because we want to achieve not only the greatest stroke, but also minimized the necessary force applied by the surgeon depressing the handle to use, the most important constraint based on the pedaling force Fš defined by equation (1). This should be due to the ergonomic needs of smaller than $350 \mathrm{~N}$, and must always take positive values. From a structural viewpoint are limited to diameters of all the wheels, which must be less than $600 \mathrm{~mm}$, which is the desired starting position of the operating table plate.[20] Next, we introduce a limit for the angle $\beta$, which should be in all positions pedal crank 2 is greater than $0^{\circ}$. The resulting vector constraints, we can thus be

Tab. 3: Optimizing results. defined as:

$c=\left[\begin{array}{l}F_{\check{s}}<350, r_{4 v}<600, r_{4 m}<600 \\ r_{6 v}<600, r_{6 m}<600,-\beta<0\end{array}\right]$

The best results for the lift we got up after several days of debugging primary ingrained itself and setting optimization. The results of the optimization of values for initial zeroing $l_{k}=706 \mathrm{~mm}, l_{0}=256 \mathrm{~mm}$, $l_{1}=800 \mathrm{~mm}, l_{2}=20 \mathrm{~mm}, r_{4 v}=240 \mathrm{~mm}, r_{4 m}=71 \mathrm{~mm}$, $r_{6 v}=73 \mathrm{~mm}, r_{6 m}=51 \mathrm{~mm}$ table shows.

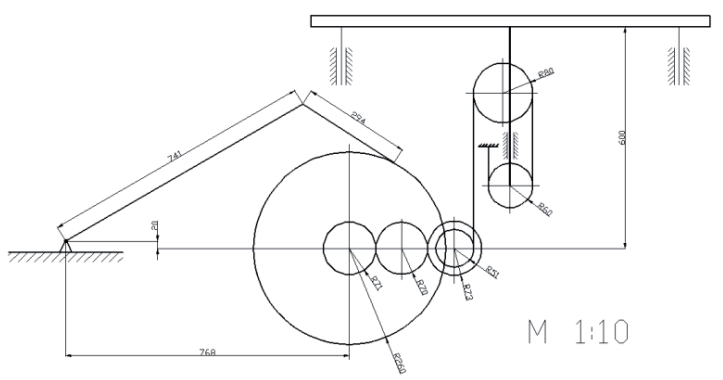

Fig. 6: Displaying final solutions in 1:10.

\section{Discussion}

The proposed solution works with reducing pedal force, and this is achieved using a gearing system of gears. Instead erektiv chain we used a system of pulleys. The advantages of this solution is to disperse the load thanks to the linseed

\begin{tabular}{|c|c|c|c|c|c|c|c|}
\hline \multicolumn{7}{|c|}{ The Resulting Parameters Mechanism [mm] } \\
\hline$l_{k}$ & $l_{o}$ & $l_{1}$ & $l_{2}$ & $r_{4 v}$ & $r_{4 m}$ & $r_{6 v}$ & $r_{6 m}$ \\
\hline 741 & 294 & 768 & 20 & 260 & 71 & 72 & 51 \\
\hline \multicolumn{3}{|c|}{$\mathrm{F}_{\mathrm{s}}$ at $\gamma=30^{\circ}[\mathrm{N}]$} & \multicolumn{3}{c|}{$\mathrm{F}_{\mathrm{s}}$ at $\gamma=10^{\circ}[\mathrm{N}]$} \\
\hline
\end{tabular}


transfer. This leads to a significant reduction in the required pedal force and efficiency pulleys. The disadvantages may be the use steel cables that can vibrate when you stop lifting. This alternative proved to be better in terms of efficiency and ease for design modifications. Even though it would be more appropriate than in surgical practice and thinking rather of using veterinary practice where the carrying capacity of the table is significantly smaller and thus the mechanism of stroke showed better qualities and also meets the criteria for pedal power

\section{Conclusions}

It has been proposed lifting mechanism operating table, which is based only on mechanical principles. The proposal was subjected secondly kinematic analysis and synthesis, and was described in detail. Furthermore, relations were determined to force conditions which govern the operation of pedaling on the pedal and relationships for stroke, which occurs due to pedaling. Finally, it has been described procedure design solutions and the possible problems that could occur during operation could. It also described the issue of optimization with constraints and Algorithms that minimizing respectively maximizing use. For your best features, a little time-consuming and good convergence was chosen interior point algorithm by which the parameters were optimized individual members mechanisms and maximized stroke. Further been set restrictive conditions that are specific mechanism design and requirements for the minimum required pedal force. Concept are found as optimal for veterinary and terrain usage. It is hard to declare which of the proposed mechanism is more appropriate. The author would tend to recommend the mechanism based on the erective chain because of his rigidity.

\section{Acknowledgments \\ The work and the contributions were supported by the project SV4506631/2101 'Biomedical Engineering Systems XII'.}

\section{References}

[1] M. Y. Lv, Z. Wu, and W. L. Qu, Shaking table test on ship lifts model with roof MR damper isolation system, 2004.

[2] B. Zipperer, "ELEVATING PLATFORMS AND LIFTING TABLES FOR PAPER INDUSTRY," Papier, vol. 24, pp. 517-\&, 1970.

[3] H. Jahsman and G. Menor, "Medical couch incorporating a lifting mechanism," ed: Google Patents, 1984.

[4] L. Guthova, L. Bednarcikova, M. Michalikova, and J. Zivcak, "Stability Evaluation of the Elderly Using a Force Plate," in 6th European Conference of the International Federation for Medical and Biological Engineering. vol. 45, I. Lackovic and D. Vasic, Eds., ed, 2015, pp. 305-308.

[5] R. Hudak, I. Polacek, L. Fedorova, P. Halfarova, M. Lisy, and J. Zivcak, "Mechanical Properties of Lumbar Bilateral Systems Using Two Different Spinal Rods are Comparable," in 6th European Conference of the International Federation for Medical and Biological Engineering. vol. 45, I. Lackovic and D. Vasic, Eds., ed, 2015, pp. 285-289.

[6] V. Rajtukova, R. Hudak, J. Zivcak, P. Halfarova, and R. Kudrikova, "Pressure Distribution in Transtibial Prostheses Socket and the Stump Interface," in Modelling of Mechanical and Mechatronic Systems. vol. 96, F. Trebuna, Ed., ed, 2014, pp. 374-381.

[7] V. Rajt'ukova, M. Michalikova, L. Bednarcikova, A. Balogova, and J. Zivcak, "Biomechanics of Lower Limb Prostheses," in Modelling of Mechanical and Mechatronic Systems. vol. 96, F. Trebuna, Ed., ed, 2014, pp. 382-391.

[8] T. Edmo and P.-O. Sehlstedt, "Lifting table," ed: Google Patents, 1986.

[9] J. Majernik, M. Dziakova, J. Zivcak, and leee, Utilization of Whole Body Vibration in Therapy of Patients with Neurological Disorders, 2013.

[10] J. Majernik, M. Dziakova, J. Zivcak, and leee, "Rehabilitation in Children with Neurological Disorders Using Whole Body Vibration," 2015 International Conference on Information and Digital Technologies (Idt), pp. 235-238, 2015.

[11] J. Majernik, M. Molcan, J. Zivcak, and leee, "Quantification of Postural Stability Changes in Patients with Impairments in Postural Control," 2014 10th International Conference on Digital Technologies (Dt), pp. 230-234, 20142014.

[12] J. Majernik and J. Zivcak, "Evaluation of Postural Stability Using Motion Analysis Techniques," in Applications of Computational Intelligence in Biomedical Technology. vol. 606, R. Bris, J. Majernik, K. Pancerz, and E. Zaitseva, Eds., ed, 2016, pp. 175-184.

[13] W.-C. Wang, C.-C. Cheng, R.-T. Chen, and Y.-W. Hsu, "Venetian blind lifting mechanism provided with concealed pull cords," ed: Google Patents, 2000.

[14] B. E. McCrimmon, "Lifting mechanism and health care equipment that incorporates the lifting mechanism," ed: Google Patents, 2008.

[15] R. M. Carroll, "Examination chair with lifting and tilting mechanism," ed: Google Patents, 2000.

[16] J. Zivcak, D. Simsik, E. Kahancova, I. Soc, and J. Control Engineers, "REHABILITATIVE AND DIAGNOSTIC ROBOT SYSTEM FOR UPPER EXTREMITIES," Ismcr 92 : Proceedings of the Sec- 
ond International Symposium on Measurement and Control in Robots, pp. 543-548, 1992.

[17] J. Zivcak, M. Uljaky, Z. Sobotova, and M. Dankovic, Manipulation system for the reposition and fixation of bones at fractures, 1998.

[18] I. S. Jahan, M. Prilepok, V. Snasel, and M. Penhaker, "Similarity analysis of EEG data based on self organizing map neural network," Advances in Electrical and Electronic Engineering, vol. 12, pp. 547-556, 2014

[19] S. T. Coates, "Patient lifting table," ed: Google Patents, 1985.

[20] O. Frantisek, "Analýza a optimalizace mechanismu řazení," Applied Mechanics, VSB-TU Ostrava, Ostrava, 2008.

\section{Biographical notes}

Lukas Novak, Ing finished MSci in 2010 at Faculty of Mechanics at VŠB Technical University of Ostrava, Czech Republic.

Marek Penhaker, Doc. Ing, Ph.D. finished MSci in 1996 at Faculty of Electrical Engineering and Computer science in specialization Measurement and Control in Biomedicine at VŠB - Technical University of Ostrava, Czech Republic. He received Ph.D. in Technical Cybernetics from VŠB - TU Ostrava. In 2016 he achieved assistant professor at Czech technical University in Prague. The field of work is biosignals measurement, transmission and processing. Through his career he published more than 130 original research articles including over 30 peer reviewed journal papers. He is author and coauthor of more than fifteen books. He realized more than 30 patents and utility models and prototypes.

Ondrej Frantisek, Ing. ,Ph.D. finished MSci in 2001 at Faculty of Mechanics at VŠB - Technical University of Ostrava, Czech Republic. He received Ph.D. in Mechanics from VŠB - TU Ostrava. The field of work is mechanics applied, optimizing, Through his career he published more than 30 original research articles including over 10 peer reviewed journal papers. 